\title{
Increased risk of posterior circulation infarcts among ischemic stroke patients with cervical spondylosis
}

\author{
Chih-Chi Chen' \\ Chia-Ying Chung' \\ Tsong-Hai Lee ${ }^{2}$ \\ Wei-Han Chang' \\ Simon FT Tang' \\ Yu-Cheng Pei ${ }^{1,3}$ \\ 'Department of Physical Medicine \\ and Rehabilitation, Chang Gung \\ Memorial Hospital, Chang Gung \\ University, College of Medicine, \\ ${ }^{2}$ Department of Neurology, Chang \\ Gung Memorial Hospital, Chang \\ Gung University, College of Medicine, \\ ${ }^{3}$ Healthy Aging Research Center, \\ Chang Gung University, Taoyuan, \\ Taiwan
}

Correspondence: Tsong-Hai Lee Stroke Center, Department of Neurology, Chang Gung Memorial Hospital, Linkou Medical Center, Chang Gung University, College of Medicine, 5, Fu-Hsing St, Kweishan, Taoyuan, 333 Taiwan

Email thlee@adm.cgmh.org.tw

Yu-Cheng Pei

Department of Physical Medicine and Rehabilitation, Chang Gung Memorial Hospital, Linkou Medical Center, Chang Gung University, College of Medicine, 5, Fu-Hsing St, Kweishan, Taoyuan, 333 Taiwan

Email yspeii@adm.cgmh.org.tw
This article was published in the following Dove Press journal:

Neuropsychiatric Disease and Treatment

3 February 2015

Number of times this article has been viewed

Background: Cervical spondylosis is one of the extrinsic factors causing vertebral artery stenosis. Several case studies have reported compression of the vertebral artery induced by cervical osteophytes that has resulted in posterior circulation infarcts (POCI). However, to the best of our knowledge, no studies have yet analyzed differences in the risk factors and stroke subtypes between ischemic stroke patients with cervical spondylosis and those without.

Purpose: In the case-controlled study reported here, we analyzed the risk factors and stroke subtypes in ischemic stroke patients with and without cervical spondylosis. Characteristics in all the recruited patients with POCI and non-POCI were further compared to extract other risk factors that could predict the occurrence of POCI.

Methods and patients: We filtered out ischemic stroke patients with cervical spondylosis ("Stroke+C" group) by International Classification of Diseases, Ninth Revision codes. We analyzed the data of 38 subjects in the Stroke+C group and 152 sex- and age-comparable ischemic stroke patients without cervical spondylosis ("Stroke-C" group). We recorded the demographic characteristics including sex and age, and stroke risk factors, including diabetes mellitus, hypertension, heart disease, hyperlipidemia, and smoking habits. The stroke classifications were defined by the Oxford Community Stroke Project classification. All subjects were further categorized into POCI or non-POCI groups. The ultrasound findings of the vertebral arteries (extracranial and intracranial) in the Stroke+C group were also recorded.

Results: More patients in the Stroke+C group tended to have POCI $(34.2 \%)$ than patients in the Stroke-C group (17.5\%) (odds ratio [OR] $=2.41, P<0.05$ ). Furthermore, hypertension $(\mathrm{OR}=3.41, P<0.01)$ and cervical spondylosis $(\mathrm{OR}=2.41, P<0.05)$ were two independent risk factors for POCI in ischemic stroke patients.

Conclusion: Ischemic stroke patients with cervical spondylosis are more prone to POCI than those without cervical spondylosis. Hypertension is another identified risk factor for POCI in ischemic stroke patients. The occurrence of POCI should be highlighted for patients with cervical spondylosis.

Keywords: risk factors, vertebral artery stenosis, cervical osteophytes

\section{Introduction}

"Cervical spondylosis" is defined as degenerative changes of the cervical spine, which often start in the intervertebral discs with osteophyte formation and involvement of adjacent soft tissue structures. ${ }^{1}$ Besides the commonly known complications of cervical spondylosis, such as pain, radiculopathy, and myelopathy, ${ }^{1}$ cervical spondylosis may also compromise the vertebral arteries extramurally, especially during head turning and extension, and is thus an extrinsic factor causing vertebral artery stenosis., ${ }^{2,3}$ Vertebral artery stenosis may then induce vertebrobasilar insufficiency 
(VBI) or posterior circulation infarcts (POCI). When VBI or POCI occur as head rotation within normal physiologic range, they are called "bow hunter's syndrome", also referred to as "bow hunter's stroke" or "rotational vertebral artery occlusion syndrome". ${ }^{4}$

Several case studies have reported that the compression of a vertebral artery induced by cervical osteophytes resulted in VBI or POCI. ${ }^{2,5-11}$ Dizziness is the most common symptom which may related to the fact that vascular supply to the vestibulocochlear organs is the end artery of the vertebrobasilar system and is more susceptible to compression-induced ischemia. ${ }^{3,12}$ However, as far as we are aware, no studies have yet analyzed if there are differences in risk factors and stroke subtypes between ischemic stroke patients with cervical spondylosis and those without. Finding out the differences may give us some information about the relationship between cervical spondylosis and ischemic stroke.

In this case-controlled study, we analyzed the risk factors and stroke subtypes in ischemic stroke patients with and without cervical spondylosis. Characteristics in all the recruited patients with POCI and non-POCI were further compared to extract other risk factors that could predict the occurrence of POCI.

\section{Materials and methods}

The study was approved by the Institutional Review Board of Institutional Review Board (IRB) of the Chang Gung Memorial Hospital, Taoyuan, Taiwan. We conducted a retrospective case-control review of adult patients with ischemic stroke from January 2000 to December 2009 in a medical center in Taiwan. Specifically, the target patients were those who had both ischemic stroke and cervical spondylosis; those recruited became the "Stroke $+\mathrm{C}$ " group. We then recruited other sex- and age-matched ischemic stroke patients without cervical spondylosis ("Stroke-C" group) as control patients and the ratio between the target and control patients was set as 1:4. Cases in Stroke+C and Stroke-C groups were filtered via the International Classification of Diseases, Ninth Revision (ICD-9) codes. The ICD-9 codes used to identify ischemic stroke were 433-437 while excluding patients without mention of cerebral infarcts $(43300,43310,43320$, 43330, 43380, 43390, 43400, 43410, and 43490). The ICD-9 codes used to identify patients with cervical spondylosis were 7210, 7211, 7230, 7222, 7224, 72270, 72271, 7226, 72290 , and 72291 . The dataset was limited to patients older than 18 years of age.

The medical records were reviewed manually. Stroke was diagnosed according to the definition of the World Health
Organization (WHO) as a clinical syndrome characterized by rapidly developing clinical symptoms or signs, and at times global loss of cerebral function, with symptoms lasting more than 24 hours or leading to death, with no apparent cause other than a vascular one and confirmed by at least one neurologist. ${ }^{13}$ Ischemic stroke was further confirmed by brain-imaging study with no evidence of intracranial hemorrhage. Cervical spondylosis was diagnosed according to the patient's cervical plain film, computed tomography, or magnetic resonance imaging findings. The exclusion criteria for Stroke+C group were: not fulfilling the WHO definition of ischemic stroke, no image confirmation of cervical spondylosis, a history of previous stroke, concomitant other central nervous disease, past head or cervical spine trauma, and/or cervical spine operation; the exclusion criteria for Stroke-C group were the same besides a diagnosis for cervical spondylosis.

\section{Medical record review}

We recorded the demographic characteristics, including sex and age, and stroke risk factors, including "diabetes mellitus" (defined as receiving medication for diabetes mellitus, fasting blood sugar $\geq 126 \mathrm{mg} / \mathrm{dL}$, or 2-hour postprandial blood sugar $\geq 200 \mathrm{mg} / \mathrm{dL}$ ), "hypertension" (defined as receiving medication for hypertension or blood pressure $>140 / 90 \mathrm{mmHg}$ on repeated measurements), heart disease (coronary heart disease, arrhythmia, heart failure, or valvular heart disease), "hyperlipidemia" (defined as receiving a cholesterol or triglyceride-reducing agent, or an overnight fasting cholesterol $>200 \mathrm{mg} / \mathrm{dL}$, or low-density lipoprotein $\geq 130 \mathrm{mg} / \mathrm{dL}$ ), and smoking habits (current smoker or a patient who had quit smoking $<$ half year). Stroke classification followed the Oxford Community Stroke Project (OCSP) classification, which categorizes ischemic stroke into total anterior circulation infarcts (TACI), partial anterior circulation infarcts (PACI), lacunar infarcts (LACI), and posterior circulation infarcts (POCI). ${ }^{14}$ All subjects were classified into either a POCI group or non-POCI group. The ultrasound findings of the vertebral arteries (extracranial and intracranial) in Stroke $+\mathrm{C}$ group were also recorded.

\section{Statistics}

Statistical analysis was performed using SPSS software (v 20.0, IBM Corporation, Armonk, NY, USA). Pearson's chi-square test or Fisher's exact test were used to compare the frequency of categorical variables between the Stroke $+\mathrm{C}$ and Stroke-C groups, and between the POCI and non-POCI groups. Student's $t$-test and the Mann-Whitney test were used 


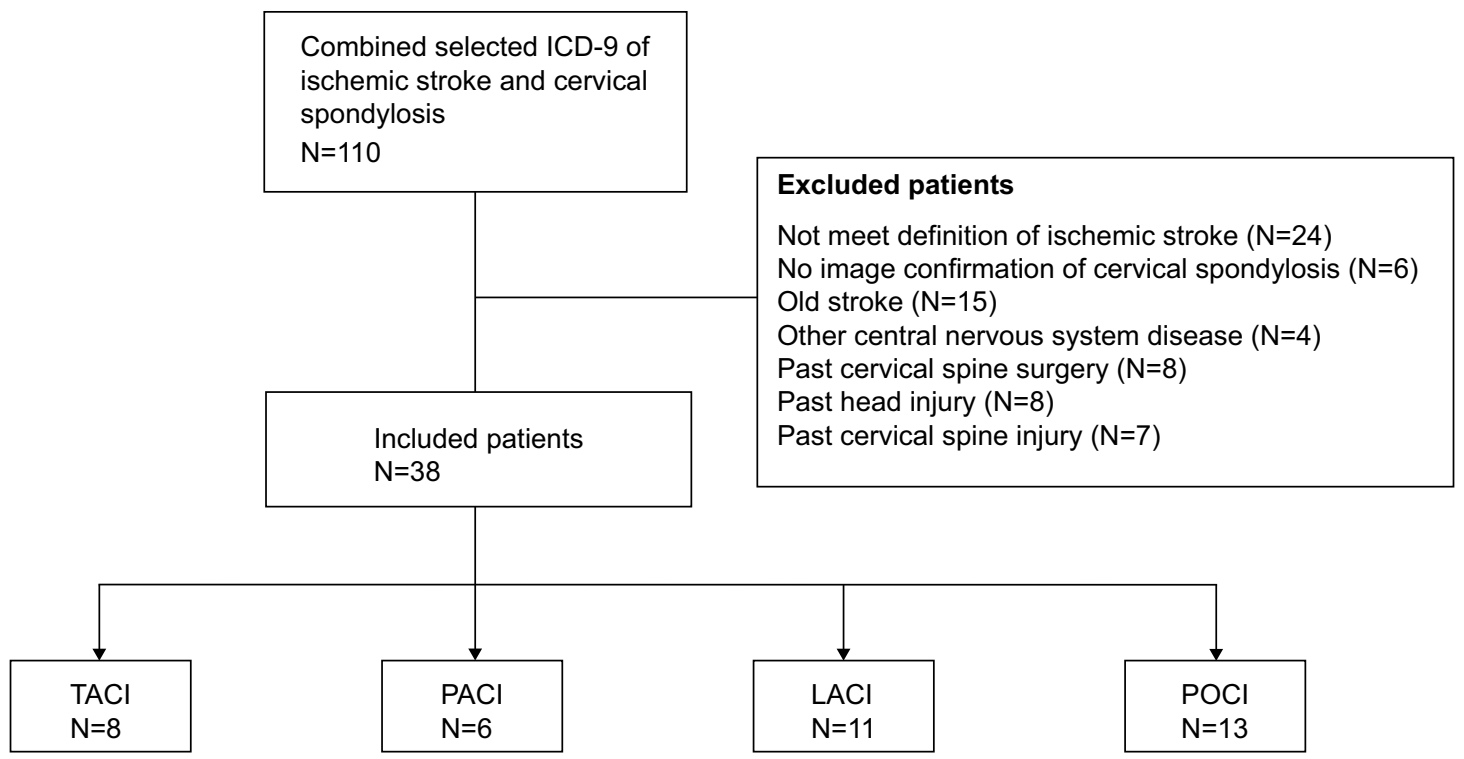

Figure I Flow chart of patient selection for stroke and cervical spondylosis.

Abbreviations: ICD-9, International Classification of Diseases, Ninth Revision; LACI, lacunar infarcts; PACl, partial anterior circulation infarcts; POCI, posterior circulation infarcts; $\mathrm{TACl}$, total anterior circulation infarcts.

to compare continuous variables between groups. Univariate and multivariate stepwise analyses were calculated by logistic regression in the case of qualitative variables. A $P$-value $<0.05$ was considered statistically significant.

\section{Results}

A total of 110 patients were first filtered out for combined diagnosis of ischemic stroke and cervical spondylosis. After checking against the inclusion and exclusion criteria, 38 cases were eventually identified for the Stroke+C study group (Figure 1). Among those 38 patients, nine were women (23.7\%) and 27 were men (76.3\%). Their mean age was 67.5 (range 46 to 84 ) years. Another 152 age- and sex-matched ischemic stroke patients without cervical spondylosis were enrolled into the Stroke-C group.

\section{Ischemic stroke patients with and without cervical spondylosis}

A comparison of the demographic data and risk factors of the Stroke+C and Stroke-C groups is shown in Table 1.

Table I Characteristics and risk analysis of ischemic stroke patients with and without cervical spondylosis

\begin{tabular}{|c|c|c|c|c|}
\hline Characteristic & Stroke $+\mathbf{C}(\mathbf{N}=\mathbf{3 8})$ & Stroke-C $(N=\mid 52)$ & Odds ratio $(95 \% \mathrm{Cl})$ & $P$-value \\
\hline Age, mean \pm SD & $67.5 \pm 9.1$ & $65.9 \pm 12.0$ & & 0.97 \\
\hline \multicolumn{5}{|l|}{ Risk factors, n (\%) } \\
\hline Hypertension & $22(57.9)$ & $98(64.5)$ & $0.76(0.37-1.56)$ & 0.46 \\
\hline Diabetes mellitus & $9(23.7)$ & $53(34.9)$ & $0.58(0.24-1.30)$ & 0.25 \\
\hline Dyslipidemia & $7(18.4)$ & $26(17.1)$ & $1.09(0.44-2.75)$ & 0.81 \\
\hline Heart disease & $7(18.4)$ & $39(25.7)$ & $0.65(0.27-1.6 I)$ & 0.40 \\
\hline Smoke & $14(36.8)$ & $51(33.6)$ & $1.16(0.55-2.42)$ & 0.71 \\
\hline Alcohol consumption & $12(31.6)$ & $26(17.1)$ & $2.24(1.00-5.00)$ & 0.07 \\
\hline \multicolumn{5}{|l|}{ OCSP classification n (\%) } \\
\hline $\mathrm{TACl}$ & $8(2 I . I)$ & $43(28.3)$ & & 0.10 \\
\hline $\mathrm{PACl}$ & $6(15.8)$ & $42(27.6)$ & & \\
\hline $\mathrm{LACl}$ & II (28.9) & $40(26.3)$ & & \\
\hline $\mathrm{POCl}$ & $13(34.2)$ & $27(17.8)$ & & \\
\hline \multicolumn{5}{|l|}{ Stroke subtype n (\%) } \\
\hline $\mathrm{POCl}$ & $13(34.2)$ & $27(17.8)$ & $2.4 \mathrm{I}(1.09-5.30)$ & 0.04 \\
\hline Non-POCl & $25(65.8)$ & $125(82.2)$ & & \\
\hline
\end{tabular}

Abbreviations: $\mathrm{Cl}$, confidence interval; $\mathrm{LACl}$, lacunar infarcts; OCSP, Oxford Community Stroke Project; PACl, partial anterior circulation infarcts; POCI, posterior circulation infarcts; Stroke-C, ischemic stroke patients without cervical spondylosis; Stroke+C, ischemic stroke patients with cervical spondylosis; TACI, total anterior circulation infarcts. 
A significantly higher incidence of POCI was found in the Stroke+C (34.2\%) group than in the Stroke-C group (17.8\%) (odds ratio $[\mathrm{OR}]=2.41, P<0.05$ ). There were no significant differences between the two groups in other demographic data or risk factors.

\section{Stroke patients with posterior circulation infarcts and non-posterior circulation infarcts}

Of the 190 recruited patients, 40 (21.1\%) had POCI. The demographic data and risk factors for the POCI and nonPOCI groups are shown in Tables 2 and 3. There was a higher incidence of hypertension in the POCI group (82.5\%) than in the non-POCI group $(58.0 \%)(\mathrm{OR}=3.41, P<0.01)$. Analogous to the result shown in Table 1, a higher incidence of cervical spondylosis was found in the POCI group (32.5\%) than in the non-POCI group $(16.7 \%)(\mathrm{OR}=2.41, P<0.05)$. No significant differences were found for age, sex, or other stroke risk factors between the POCI and non-POCI groups (Table 2).

It was then important to examine whether cervical spondylosis and hypertension are both significant predictors for the occurrence of POCI and if there is any confounding between them. Thus, we conducted a multivariable logistic regression analysis using POCI as the dependent variable, and found that hypertension and cervical spondylosis are two significant and independent determinants for POCI (Table 3).

\section{Ultrasound findings of vertebral arteries in ischemic stroke patients with cervical spondylosis}

Among the 38 patients in Stroke+C group, five had ultrasound data missing. Two ultrasound findings identified hypoplasia

Table 2 Characteristics and risk factors for ischemic stroke patients with posterior circulation infarcts and non-posterior circulation infarcts

\begin{tabular}{llll}
\hline Characteristic & $\begin{array}{l}\text { POCI } \\
(\mathbf{N}=\mathbf{4 0})\end{array}$ & $\begin{array}{l}\text { Non-POCI } \\
(\mathbf{N}=150)\end{array}$ & P-value \\
\hline Age, mean \pm SD & $67.5 \pm 9.1$ & $65.9 \pm 12.0$ & 0.97 \\
Sex, male (\%) & $27,67.5 \%$ & $1 \mathrm{I} 8,78.7 \%$ & 0.15 \\
Risk factor, $\mathbf{n}(\%)$ & & & \\
Hypertension & $33(82.5)$ & $87(58.0)$ & 0.004 \\
Diabetes mellitus & $15(37.5)$ & $47(31.3)$ & 0.46 \\
Dyslipidemia & $8(20)$ & $25(16.7)$ & 0.64 \\
Heart disease & $9(22.5)$ & $37(24.7)$ & 0.84 \\
Smoker & $13(32.5)$ & $52(34.7)$ & 0.85 \\
Alcohol & $6(15)$ & $32(21.3)$ & 0.51 \\
Cervical spondylosis & $13(32.5)$ & $25(16.7)$ & 0.04 \\
\hline
\end{tabular}

Abbreviations: Non- $\mathrm{POCl}$, non-posterior circulation infarcts; $\mathrm{POCl}$, posterior circulation infarcts.
Table 3 Risk factors for ischemic stroke patients with posterior circulation infarct versus non-posterior circulation infarct groups using multivariable logistic regression analysis

\begin{tabular}{llll}
\hline Risk factor & \multicolumn{3}{l}{ Multivariate analysis } \\
\cline { 2 - 4 } & Odds ratio & $\mathbf{9 5 \%} \mathbf{C l}$ & $\mathbf{P}$-value \\
\hline Hypertension & $3.4 \mathrm{I}$ & $\mathbf{1} .42-8.2 \mathrm{I}$ & 0.004 \\
Diabetes mellitus & $\mathrm{I} .32$ & $0.64-2.72$ & 0.68 \\
Dyslipidemia & $\mathrm{I} .25$ & $0.5 \mathrm{I}-3.03$ & 0.93 \\
Heart disease & 0.89 & $0.39-2.03$ & 0.70 \\
Smoker & $0.9 \mathrm{I}$ & $0.43-1.9 \mathrm{I}$ & 0.45 \\
Alcohol consumption & 0.65 & $0.25-1.68$ & 0.23 \\
Cervical spondylosis & $2.4 \mathrm{I}$ & $\mathrm{I} .09-5.30$ & $0.0 \mathrm{I}$ \\
\hline
\end{tabular}

Abbreviation: $\mathrm{Cl}$, confidence interval.

of one side of the vertebral artery. Five ultrasounds identified vertebral artery stenosis, among which four were extracranial while one was intracranial. Among the 13 patients with POCI in the Stroke+C group, two had ultrasound data missing. Three of the other eleven patients $(27 \%)$ were identified as having extracranial vertebral artery stenosis.

\section{Discussion}

As far as we are aware, this study has produced the largest amount of clinical data to date in support of ischemic stroke patients with cervical spondylosis being more prone to having POCI than those without cervical spondylosis. An OR of 2.41 indicates a stronger predictive value from a clinical perspective. The incidence of POCI in the recruited patients is comparable to that reported in previous studies. ${ }^{15,16}$ Generally, POCI contributes to about one quarter of all ischemic stroke.

The causes of vertebral artery stenosis are classified as intrinsic or extrinsic lesions. Intrinsic lesions include atherosclerosis, vasculitis, and dissection, while extrinsic lesions include neoplasm, osteophyte, fibrous bands, discs, spinal fracture/dislocation, and infection. ${ }^{7,8}$ Although case reports and case series have discussed the causality between cervical spondylosis and VBI/POCI, 5,7-9,11,17 the reported case number was relatively limited especially for those with POCI. Denis et al reviewed the reported surgical cases of vertebral artery compression caused by cervical spondylosis by searching PubMed and found 49 published cases. ${ }^{9}$ Among them, only nine cases in three reports had infarcts ${ }^{7,18,19}$ and the other 40 cases were transient ischemic attacks. Recently, Zaidi et al reported their experiences in diagnosing and managing of bow hunter's syndrome over 15 years. ${ }^{4}$ They reported eleven cases, only one of which had POCI. The small case number could contribute to the fact that extracranial vertebral artery lesions could be asymptomatic unless bilateral vertebral 
arteries were compromised. ${ }^{7,20}$ Furthermore, vertebral artery occlusion due to cervical spondylosis compression may not be easily confirmed unless the patient undergoes dynamic angiography or dynamic computed tomographic angiography as the patient rotates or extends the neck. ${ }^{11,17}$

Cervical osteophytes arising from the uncinate process or the facet complex could be an extrinsic factor for vertebral artery stenosis, as these would compress the vertebral artery extramurally. ${ }^{2}$ The second segment of vertebral artery enters the foramen transversarium of the sixth cervical vertebra and exists from the foramen transversarium of the second cervical vertebra. The most frequently involved levels of cervical osteophytes that induce vertebral artery incompetence are the fifth and sixth (56\%), the fourth and fifth (24\%), and the sixth and seventh (20\%) levels. ${ }^{8,17}$ The compression may be exacerbated by neck rotation to the contralateral side or neck extension. ${ }^{9}$ Machaly et $\mathrm{a}^{12}$ found that patients with cervical spondylosis and vertigo would experience a decrease of blood flow in both vertebral arteries when their head is rotated, a finding that further supports a cervical cause of POCI.

This study found that patients in the Stroke $+\mathrm{C}$ group had a significantly higher incidence of POCI than those in the Stroke-C group. In this study, $27 \%$ of POCI subjects in Stroke $+\mathrm{C}$ group were identified as having vertebral artery stenosis by Doppler ultrasound examination in the neutral position; such incidence is similar to that found in previous study, which reported that about $20 \% \sim 25 \%$ of POCI is induced by vertebral artery stensosis. ${ }^{16}$ The reason there was no obvious elevated vertebral artery stenosis in the Stroke $+C$ group may be due the fact that vertebral artery compromise in different head positions, especially rotation and/or extension, may be missed in standard Doppler ultrasound performed with the head in the neutral position.

As an intrinsic factor, vertebral artery stenosis is most commonly caused by diffuse atherosclerosis. ${ }^{21}$ There is a correlation between the tortuous course of the vertebral artery and the occurrence of atherosclerotic plaque in the vertebral segment. ${ }^{22}$ In the vertebral segment, the artery is in close relationship medially with the uncinate process of each vertebral body and posteriorly with the facet complex. The uncinate process is the most common site of osteophytosis, which may partially cover the underlying transverse foramen from an anteromedial direction. Covering of the foramen by osteophyte from the zygapophyseal joints is less frequent. ${ }^{6}$ It has been hypothesized that the frequent changes of the direction of the vertebral artery around the prominent osteophytes may increase the risk of atherosclerosis for the increased tortuous course. ${ }^{23}$ Besides, rapid and marked stretching of the arteries when crossing osteophytes can cause vasospasm, arterial dissection, or intramural hemorrhage. These repeated microtraumas lead to thrombi, atherosclerosis, and decreased blood flow in the vessels. ${ }^{24}$

In order to find out other risk factors that may predispose stroke patients to POCI and confound results, we compared the stroke risk factors between POCI and non-POCI in all recruited patients. We found that besides cervical spondylosis, hypertension is also a significant risk factor for POCI. Such findings agree with previous studies in that hypertension was present more often in patients with posterior circulation stroke than in those with anterior circulation stroke. ${ }^{25,26}$ Hypertension is a well known risk factor for the development of atherosclerosis. ${ }^{27}$ The subsequent multivariate analysis was undertaken to see if there was any confounding between cervical spondylosis and hypertension, and the results identified these as two independent risk factors for POCI in ischemic stroke patients.

\section{Limitations}

The present study has several limitations. First, for a retrospective study, selection bias is inevitable, even when age and sex are matched. Second, the results are based on a hospital-based registry, limiting the variety of the sampled population. Third, patients in the control group may also have had subclinical cervical spondylosis, since cervical spondylosis is common in the elderly. Fourth, all the patients with infarcts only underwent color duplex sonography of the vertebral artery with their head in the neutral position. The flow may not be compromised without head rotation and/or extension. In this sense, further prospective cohort studies may be necessary to strengthen the causality between cervical spondylosis and POCI.

\section{Conclusion}

Cervical spondylosis is a risk factor for POCI. Besides cervical spondylosis, hypertension is another identified risk factor for POCI. Clinicians should keep in mind that, besides the common complications such as pain, radiculopathy, and myelopathy, cervical spondylosis could also predispose to POCI.

\section{Disclosure}

The authors declare no conflicts of interest in this work.

\section{References}

1. Binder AI. Cervical spondylosis and neck pain. BMJ. 2007;334(7592): 527-531.

2. George B, Laurian C. Impairment of vertebral artery flow caused by extrinsic lesions. Neurosurgery. 1989;24(2):206-214. 
3. Owolabi MO, Ogah OS, Ogunniyi A. Episodic vertigo resulting from vascular risk factors, cervical spondylosis and head rotation: Two case reports. Neuropsychiatr Dis Treat. 2007;3(5):675-678.

4. Zaidi HA, Albuquerque FC, Chowdhry SA, Zabramski JM, Ducruet AF, Spetzler RF. Diagnosis and Management of Bow Hunter's Syndrome: 15-Year Experience at Barrow Neurological Institute. World Neurosurg. 2014;82(5):733-738.

5. Burneo JG, Mitsias PD. Vertebrobasilar territory ischemia due to cervical spondylosis. Cerebrovasc Dis. 2002;13(1):78.

6. Cagnie B, Barbaix E, Vinck E, D'Herde K, Cambier D. Extrinsic risk factors for compromised blood flow in the vertebral artery: anatomical observations of the transverse foramina from C3 to C7. Surg Radiol Anat. 2005;27(4):312-316.

7. Choi JM, Hong HJ, Chang SK, Oh SH. Cerebellar infarction originating from vertebral artery stenosis caused by a hypertrophied uncovertebral joint. J Stroke Cerebrovasc Dis. 2012;21(8):908.e7-908.e9.

8. Citow JS, Macdonald RL. Posterior decompression of the vertebral artery narrowed by cervical osteophyte: case report. Surg Neurol. 1999;51(5):495-498; discussion 498-499.

9. Denis DJ, Shedid D, Shehadeh M, Weil AG, Lanthier S. Cervical spondylosis: a rare and curable cause of vertebrobasilar insufficiency. Eur Spine J. 2014;23 Suppl 2:206-213.

10. Kuether TA, Nesbit GM, Clark WM, Barnwell SL. Rotational vertebral artery occlusion: a mechanism of vertebrobasilar insufficiency. Neurosurgery. 1997;41(2):427-432; discussion 432-423.

11. Mourand I, Azakri S, Boniface G, Bonafe A, Maldonado IL. Teaching NeuroImages: intermittent symptomatic occlusion of the vertebral artery caused by a cervical osteophyte. Neurology. 2013;80(5):e54.

12. Machaly SA, Senna MK, Sadek AG. Vertigo is associated with advanced degenerative changes in patients with cervical spondylosis. Clin Rheumatol. 2011;30(12):1527-1534.

13. Tunstall-Pedoe H; WHO MONICA Project principal investigators. The World Health Organization MONICA Project (Monitoring Trends and Determinants in Cardiovascular Disease): A major international collaboration. J Clin Epidemiol. 1988;41:105-114.

14. Bamford J, Sandercock P, Dennis M, Burn J, Warlow C. Classification and natural history of clinically identifiable subtypes of cerebral infarction. Lancet. 1991;337(8756):1521-1526.

15. Jeng JS, Chung MY, Yip PK, Hwang BS, Chang YC. Extracranial carotid atherosclerosis and vascular risk factors in different types of ischemic stroke in Taiwan. Stroke. 1994;25(10):1989-1993.
16. Khan S, Cloud GC, Kerry S, Markus HS. Imaging of vertebral artery stenosis: a systematic review. J Neurol Neurosurg Psychiatry. 2007; 78(11):1218-1225.

17. Bulsara KR, Velez DA, Villavicencio A. Rotational vertebral artery insufficiency resulting from cervical spondylosis: case report and review of the literature. Surg Neurol. 2006;65(6):625-627.

18. Sullivan HG, Harbison JW, Vines FS, Becker D. Embolic posterior cerebral artery occlusion secondary to spondylitic vertebral artery compression. Case report. J Neurosurg. 1975;43(5):618-622.

19. Takeuchi S, Kawaguchi T, Nakatani M, Isu T. Hemorrhagic infarction originating from vertebral artery stenosis caused by an osteophyte at the C5 superior articular process. Neurol Med Chir (Tokyo). 2009;49(3): 114-116.

20. Liff JM, Labovitz D, Robbins MS. Profound gastroparesis after bilateral posterior inferior cerebellar artery territory infarcts. Clin Neurol Neurosurg. 2012;114(6):789-791.

21. Caplan LR, Wityk RJ, Glass TA, et al. New England Medical Center Posterior Circulation registry. Ann Neurol. 2004;56(3):389-398.

22. Haneline MT, Rosner AL. Comments about "Atherosclerosis in the vertebral artery: an intrinsic risk factor in the use of spinal manipulation? (2006) Surg Radiol Anat 28:129-134, by Cagnie B, Barbaix E, Vinck E, D'Herde K, Cambier D”. Surg Radiol Anat. 2007;29(2):185-186; author reply 187.

23. Cagnie B, Barbaix E, Vinck E, D’Herde K, Cambier D. Atherosclerosis in the vertebral artery: an intrinsic risk factor in the use of spinal manipulation? Surg Radiol Anat. 2006;28(2):129-134.

24. Akar Z, Kafadar AM, Tanriover N, et al. Rotational compression of the vertebral artery at the point of dural penetration. Case report. J Neurosurg. 2000;93(2 Supp1):300-303.

25. Miyamoto N, Tanaka Y, Ueno Y, Tanaka R, Hattori N, Urabe T. Comparison of clinical backgrounds with anterior versus posterior circulation infarcts. J Stroke Cerebrovasc Dis. 2010;19(5):393-397.

26. Kim JS, Nah HW, Park SM, et al. Risk factors and stroke mechanisms in atherosclerotic stroke: intracranial compared with extracranial and anterior compared with posterior circulation disease. Stroke. 2012;43(12):3313-3318.

27. Alexander RW. Theodore Cooper Memorial Lecture. Hypertension and the pathogenesis of atherosclerosis. Oxidative stress and the mediation of arterial inflammatory response: a new perspective. Hypertension. 1995;25(2):155-161.
Neuropsychiatric Disease and Treatment

\section{Publish your work in this journal}

Neuropsychiatric Disease and Treatment is an international, peerreviewed journal of clinical therapeutics and pharmacology focusing on concise rapid reporting of clinical or pre-clinical studies on a range of neuropsychiatric and neurological disorders. This journal is indexed on PubMed Central, the 'PsycINFO' database and CAS,

\section{Dovepress}

and is the official journal of The International Neuropsychiatric Association (INA). The manuscript management system is completely online and includes a very quick and fair peer-review system, which is all easy to use. Visit http://www.dovepress.com/testimonials.php to read real quotes from published authors. 